\title{
A tabulated real-fluid modeling approach applied to renewable dual-fuel evaporation and mixing
}

\author{
Hesham Gaballa*1,2, Sajad Jafari ${ }^{1,2}$, Angela Di-lella ${ }^{1}$, \\ Chaouki Habchi $^{1,2}$, Jean-Charles De Hemptinne ${ }^{1}$ \\ ${ }^{1}$ IFP Energies nouvelles, 1 et 4 avenue de Bois-Préau, 92852 Rueil-Malmaison, France \\ ${ }^{2}$ Institut Carnot IFPEN Transports Energies, 1 et 4 avenue de Bois-Préau, 92852 \\ Rueil-Malmaison, France \\ ${ }^{*}$ Corresponding author email: Hesham.gaballa@ifpen.fr
}

\begin{abstract}
The substitution of diesel by cleaner renewable fuels such as short-chain alcohols in dual-fuel internal combustion engines is considered an attractive solution to reduce the pollutant emissions from internal combustion engines. In this context, accurate and robust two-phase flow models taking into account the real fuel thermodynamics are required to predict the phase change and mixing processes when various fuels are injected in sub-transcritical conditions. The present study proposes an efficient Real-Fluid model (RFM) based on a two-phase fully compressible four-equation model under mechanical and thermal equilibrium assumptions and closed by a thermodynamic equilibrium tabulation approach. Compared to previous research limited to binary mixtures tabulation, the proposed tabulation approach can further handle ternary mixtures using four-dimensional (4D) tables. In this article, the RFM model has been applied to compare the evaporation and mixing of $n$-dodecane droplets in single and bi-component ambient. For the single component nitrogen ambient, the numerical results compare well with recent experiments. Finally, $\mathrm{n}$-dodecane droplets evaporation in a bi-component (nitrogen and methanol) ambient relevant to dual-fuel engines have been explored using the Cubic Plus Association (CPA) equation of state. It was found that the initial presence of methanol in the ambient strongly accelerates the mixing process.
\end{abstract}

\section{Keywords}

Real fluid model, thermodynamic tabulation, ternary mixtures, transcritical mixing, evaporation.

\section{Introduction}

The recent strict legislation related mainly to carbon dioxide $\left(\mathrm{CO}_{2}\right)$ emissions poses a new challenge finding alternative cleaner fuels to partially or totally substitute fossil fuels in internal combustion engines (ICE). Dual-fuel internal combustion engines (DFICE) is one of the recent promising solutions to diesel fuel combustion emissions. In DFICE, a primary fuel such as natural gas or short-chain alcohols is utilized with a moderate amount of pilot diesel fuel acting only as an ignition agent. A review of the different primary fuel injection strategies in dual fuel engines can be found in [31]. Moreover, Several experimental studies [11, 16, 8] have demonstrated the potential of employing primary alcohols such as methanol and ethanol as primary fuels in DFICE to reduce the soot and NOx emissions.

Fuel evaporation and mixing are significant phenomena during the fuel injection in ICE, directly impacting the combustion efficiency and resultant emissions. Indeed, several single or multicomponent liquid droplet evaporation models have been reported in the literature [6, 3, 19, 9]. However, these models predict the heat and mass transfer processes using correlations, which may require further adjustments to consider the interaction and mixing between the pilot and primary fuels in dual-fuel engines, which subsequently affect the heat transfer and vaporization rates. Therefore, highly resolved numerical simulations relevant to dual-fuel conditions are needed to quantify the interaction between the various fuels involved and serve as a reference for the development and validation of droplet evaporation models. Besides, such simulations offer an alternative for the cases that experimental techniques are not available. 
The multi-component two-phase flow involved in such engines may exhibit different thermodynamic regimes based on the local pressure, temperature, and species composition compared to the mixture's critical point. Indeed, it can not be determined from a priori-analysis whether the spatiotemporal evolution of the involved thermodynamic states is subcritical or supercritical during the entire fuel injection event. As a matter of fact, both subcritical and supercritical regimes may exist simultaneously, as discussed by [5, 1]. Thereby, two-phase flow models that can handle such multi-component mixtures accurately and robustly are required for further development of DFICE.

Several two-phase flow models have been proposed in the literature in the framework of the diffuse interface model (DIM) approach with transport equations ranging from four to seven depending on the initial equilibrium assumptions $[20,7,13,15]$. Among them, the four-equation model has been employed for cavitation and spray simulations $[4,14]$ due to its high efficiency. The main challenge in such models is estimating the phase change source term for the classical subcritical phase change and the ability to transit to a supercritical single-phase mixing regime. Several researchers $[1,10,18]$ have coupled the four-equation model with a vaporliquid equilibrium (VLE) solver to estimate the phase change source term. However, it has been proved that the direct evaluation of the VLE calculation during the simulation is computationally demanding, especially when employing a complex real-fluid equation of state (EOS) $[1,2]$. Accordingly, a more robust and efficient tabulation approach could be one remedy to the computationally demanding VLE solver.

Several contributions related to tabulated thermodynamic closure have been presented in the literature. Yi et al. [32] investigated n-dodecane droplets evaporation in transcritical conditions using a three-dimensional uniform tabulation approach based on the VLE solver developed by [1, 2] coupled with the Peng-Robinson (PR) EOS. Besides, Koukouvinis et al. [12] proposed a tabulated thermodynamic approach based on $\log _{10} P-T$ tables to investigate the high pressure/temperature injection of $n$-dodecane in ECN spray A [33] conditions. However, the previously mentioned research was limited to the tabulation of binary mixtures. Thereby, further development of a tabulation approach capable of handling ternary mixtures as those encountered in dual-fuel engines is needed.

The current work presents an extension of the binary version of the real-fluid model (RFM) [32] to a ternary version capable of handling three-component mixtures based on four dimensional (4D) tables generated by the IFPEN-Carnot thermodynamic library. The ternary version of the RFM model closed by the tabulated Cubic Plus Association (CPA) EOS is applied to explore the evaporation of $n$-dodecane droplets in a bi-component ambient (methanol+nitrogen) relevant to dual-fuel engines.

The main aim is to investigate the impact of the methanol ambient concentration on the droplet lifetime and evaporation rate compared to the single component ambient (nitrogen) case at the same ambient pressures and temperatures. Moreover, quantify the effect of the different solubilities of methanol and nitrogen on the droplet evaporation, especially at the high ambient pressures considered. Besides, the evaporation and mixing processes take place at a subcritical or supercritical thermodynamic regime. The current work aims to investigate the aforementioned points.

This paper is organized as follows. Section 2 describes the RFM model, including the set of transport equations and the tabulation approach and thermodynamic ingredients. Section 3 firstly presents a comparison of the numerical results of $n$-dodecane droplet evaporation in a pure nitrogen ambient with the experiments of [17]; secondly, n-dodecane droplet evaporation in bi-component ambient at the same temperature and pressure conditions of [17] is discussed for various methanol ambient concentrations. Finally, Section 4 presents the conclusions. 


\section{The Real fluid model (RFM)}

\subsection{Governing equations}

The two-phase flow model used in the current work is a four-equation homogeneous equilibrium model (HEM), which is fully compressible and considers multi-component in both phases, under the assumptions of thermal and mechanical equilibrium as follows,

$$
\begin{aligned}
& \frac{\partial \rho}{\partial t}+\frac{\partial \rho u_{i}}{\partial x_{i}}=0 \\
& \frac{\partial \rho Y_{m}}{\partial t}+\frac{\partial \rho Y_{m} u_{j}}{\partial x_{j}}=\frac{\partial}{\partial x_{j}}\left(\rho D \frac{\partial Y_{m}}{\partial x_{j}}\right), m=1, \ldots, N_{s}-1 \\
& \frac{\partial \rho u_{i}}{\partial t}+\frac{\partial \rho u_{i} u_{j}}{\partial x_{j}}=-\frac{\partial P}{\partial x_{i}}+\frac{\partial \tau_{i j}}{\partial x_{j}}, \tau_{i j}=\mu\left(\frac{\partial u_{i}}{\partial x_{j}}+\frac{\partial u_{j}}{\partial x_{i}}\right)-\frac{2}{3} \mu\left(\frac{\partial u_{k}}{\partial x_{k}} \delta_{i j}\right) \\
& \frac{\partial \rho e}{\partial t}+\frac{\partial \rho e u_{j}}{\partial x_{j}}=-P \frac{\partial u_{j}}{\partial x_{j}}+\tau_{i j} \frac{\partial u_{i}}{\partial x_{j}}+\frac{\partial}{\partial x_{j}}\left(\lambda \frac{\partial T}{\partial x_{j}}\right)+\frac{\partial}{\partial x_{j}}\left(\rho \sum_{m} D h_{m} \frac{\partial Y_{m}}{\partial x_{j}}\right)
\end{aligned}
$$

where $\left(\tau_{i j}\right)$ is the viscous stress tensor. $\left(\rho, u_{i}, P, T, e\right)$ are the mixture's density, velocity, pressure, temperature and specific internal energy, respectively. $\left(Y_{m}, h_{m}\right)$ are the mass fraction and specific enthalpy of species $m=\left\{1, \ldots, N_{s}\right\}$, respectively. The laminar contribution of the thermal conductivity $(\lambda)$ and the dynamic viscosity $(\mu)$ is computed by Chung et al. [21] correlations. The laminar mass diffusion coefficient is estimated using a given Schmidt number as $(D=\nu / S c)$.

\section{Tabulated thermodynamic closure}

The fully compressible multi-component two-phase flow system described above is closed by a tabulated real-fluid equation of state (EOS) such as the Peng-Robinson (PR) EOS [23] or the cubic plus association (CPA) EOS [24] adopting a thermodynamic equilibrium hypothesis. Before the simulation, the multi-component vapor-liquid equilibrium table is generated using the IFPEN-Carot thermodynamic library. The thermodynamic library performs the VLE calculation using a robust isothermal-isobaric (TP) flash [22] coupled to various real-fluid EOS, to compute the thermodynamic equilibrium density, internal energy, fluid phase state and composition as well as necessary thermodynamic derivatives as heat capacity and sound speed and the transport properties for each group of temperature, pressure and feed. The current work employs a uniform tabulation approach based on a bijective look-up table linking $(\rho, e)$ and $(T, P)$. The mixture's density $(\rho)$ and internal energy $(e)$ are computed as $\left(\rho=\sum_{p} \alpha_{p} \rho_{p}\right)$ and $\left(e=\sum_{p} \alpha_{p} \rho_{p} e_{p} / \rho\right)$, respectively; where $\left(\alpha_{p}\right)$ denotes the phase volume fraction $(p=l$ is liquid phase and $p=v$ is vapor phase). The inputs of the 3D or 4D tables for binary and ternary mixtures, respectively are the temperature $(T)$, pressure $(P)$ and species mass fraction ( $Y_{m}, m=1, N_{s}-1$, where $N_{s}$ is the total number of species). The Inverse-Distance Weighting (IDW) method [26] is used for the interpolation of the tabulated quantities of the 3D and 4D tables. This method is based on the assumption that the value of the unsampled point is the average weight of the known values in the neighborhood, and the weight is inversely proportional to the distance between the prediction location and the sampled location. A general form of finding an interpolated value $(u)$ at a given point $(x)$ based on samples $u_{i}=u\left(x_{i}\right)$ for $i=1, . ., N$ using IDW can be expressed as follows,

$$
u(x)= \begin{cases}\frac{\sum_{i}^{N} w_{i}(x) u_{i}}{\sum_{i}^{N} w_{i}(x)}, & \text { if } d\left(x, x_{i}\right) \neq 0 \text { for all } i \\ u_{i}, & \text { if } d\left(x, x_{i}\right)=0 \text { for some } i\end{cases}
$$

where $w_{i}(x)=1 / d\left(x, x_{i}\right), x$ denotes an interpolated (arbitrary) point, $x_{i}$ is an interpolating (known) point, $d$ is the given distance from the known point $x_{i}$ to the unknown point $x, N$ is the total number of known points used in the interpolation. 


\section{Results and discussion}

The current study investigates the evaporation characteristics of an n-dodecane droplet in bicomponent ambient relevant to dual-fuel configuration using the newly developed ternary version of the RFM model implemented in CONVERGE v.3 CFD code [25] used to carry out the present simulations. The test case setup and ambient operating conditions are based on the experimental work of [17], who performed a high-speed high-resolution microscopic visualizations of $n$-dodecane droplets at ambient temperatures and pressures higher than the critical point for pure n-dodecane (transcritical conditions), where individual droplets are tracked at the end of the injection event until they are completely vaporized. Firstly, the n-dodecane droplet evaporation in single component ambient (nitrogen) is computed and compared with the experiments of [17]. Secondly, the same test cases are computed with introducing methanol in the ambient so that the ambient is a mixture of methanol and nitrogen, where the methanol ambient concentration is increased progressively to investigate its impact on the droplet evaporation.

\subsection{Test case setup}

A 2D simulation setup was employed for an almost pure n-dodecane droplet with initial mass fraction of $\left(Y_{C_{12} H_{26}}=0.9999999\right.$ and $\left.Y_{N 2}=10^{-7}\right)$, initial diameter of $(60 \mu \mathrm{m})$ and initial temperature of $(363 \mathrm{~K})$. The droplet is initially moving with a velocity of $(0.2 \mathrm{~m} / \mathrm{s})$ in an quiescent ambient filled with nitrogen. The detailed information of the simulated test cases is listed in Table 1. The computational domain size in terms of the initial droplet diameter $\left(D_{d, 0}\right)$ is $\left(16 D_{d, 0}\right.$ $\left.\mathrm{x} 10 D_{d, 0}\right)$ to reduce any interference from the boundaries. The mesh size in the far-field is set to $(16 \mu \mathrm{m})$, which is reduced to $(2 \mu \mathrm{m})$ in the vicinity of the droplet. The boundaries of the computational domain are treated as adiabatic walls. The numerical solution of the transport equations is based on the PISO algorithm for pressure-velocity coupling. A second-order central difference scheme with flux limiter is used for the spatial discretization of each equation. The time discretization is achieved by the second-order Crank-Nicolson scheme for the momentum equation and the first-order Euler scheme for the rest of the equations. The time step is automatically adjusted based on a maximum CFL number of 0.4 reaching a value in the range of $\left(10^{-9} / 10^{-10} s\right)$. The carried out highly resolved simulations don't employ a subgrid scale turbulence model for the relatively low Reynolds number of the simulated test cases. It is worth to mention that the surface tension was assumed negligible in the current simulations, since the ambient conditions are relatively higher than the $\mathrm{n}$-dodecane critical point. The appropriateness of the latter assumption will be investigated in the future work.

Table 1. Test cases operating conditions based on experiments of [17]

\begin{tabular}{c|cccccc}
\hline & $T_{a m b}(K)$ & $P_{a m b}($ bar $)$ & $D_{d, 0}(\mu m)$ & $U_{d, 0}(m / s)$ & $T_{d, 0}(K)$ & $R e_{d}$ \\
\hline case 1 & 1200 & 106 & 60 & 0.2 & 363 & 7.4 \\
case 2 & 1000 & 88 & 60 & 0.2 & 363 & 8.3 \\
\hline
\end{tabular}

\subsection{Droplet evaporation in single-component ambient validation}

The RFM model thermodynamic closure for the n-dodecane/nitrogen (binary mixture) is based on the PR-EOS, with thermodynamic table resolution in $\left[P, T, Y_{C_{12} \mathrm{H}_{26}}\right]$ axes of $[21,201,101]$ points, respectively.

The temporal evolution of the normalized squared equivalent droplet diameter and the droplet shape compared with the experimental images [17] can be seen in Figure 1 for case 1 . The calculated droplet diameter is the equivalent droplet diameter, since the droplet deforms throughout its lifetime and is formulated in 2D as $\left[d=\left(\frac{4}{\pi} \sum_{\text {cells }} \alpha_{l} V_{\text {cell }}\right)^{0.5}\right]$, where $\alpha_{l}$ is the cell liquid volume fraction and $V_{\text {cell }}$ is the cell volume. Generally, a good qualitative agreement with the experiments is achieved for the droplet shape at the different time instants (Figure 1b). Moreover, the droplet lifetime based on the simulation falls within the lifetime range estimated from the published experimental video sequences (Figure 1a). 


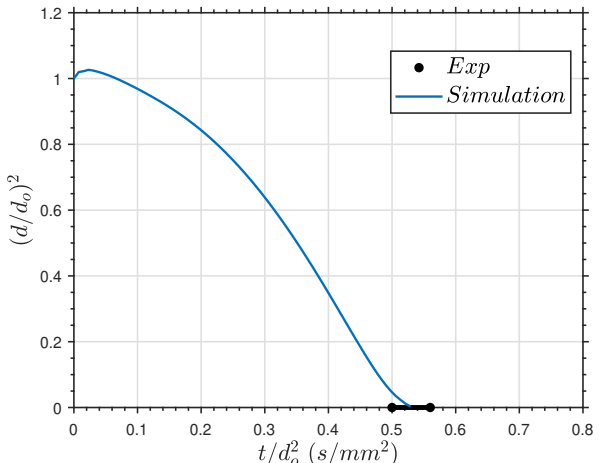

(a)

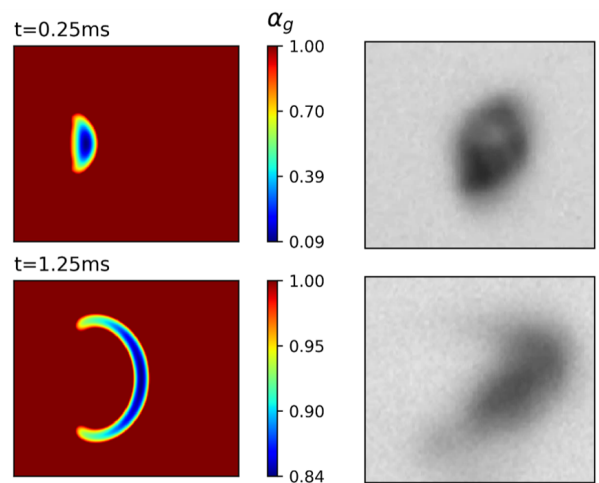

(b)

Figure 1. (a) Temporal evolution of the dimensionless squared equivalent droplet diameter compared to the droplet lifetime range estimated from the experimental video sequences [17] , (b) Comparison of the gas volume fraction $\left(\alpha_{g}\right)$ from the simulation (left) with the experimental images [17] (right) at different time instants for case 1 $\left(P_{a m b}=106 b a r, T_{a m b}=1200 K\right)$. The droplet moves from left to right.

\subsection{Droplet evaporation in bi-component ambient}

In this section, the n-dodecane droplet is evaporating in bi-component ambient (Nitrogen, $\mathrm{N}_{2}$ and methanol, $\mathrm{CH}_{3} \mathrm{OH}$ ). The same operating temperature and pressure conditions in the previous section are employed but with introducing methanol in the ambient with various initial concentrations. More precisely, the initial mass fraction of methanol in the ambient is increased from 0.2 to 0.6 with an increment of 0.2 and the initial mass fraction of nitrogen is accordingly $\left(Y_{N_{2}}=1-Y_{\mathrm{CH}_{3} \mathrm{OH}}\right)$. The RFM model thermodynamic closure for the ( $\mathrm{n}$ dodecane/nitrogen/methanol) ternary mixture is based on the CPA-EOS, with thermodynamic table resolution in $\left[P, T, Y_{C_{12} H_{26}}, Y_{N_{2}}\right]$ axes is $[21,201,101,101]$ points, respectively. Compared to cubic EOS, the CPA EOS with its additional association term can be efficiently used to model mixtures including hydrocarbons and polar compounds (methanol) [27]. The variation of the methanol ambient concentration leads to a variation in the ambient density $\left(\rho_{\infty}\right)$ and viscosity $\left(\mu_{\infty}\right)$ and accordingly the Reynolds number $\left[R e_{d}=\frac{\rho_{\infty} U_{d, r e l} D_{d, 0}}{\mu_{\infty}}\right]$ increases for the same initial drop diameter and velocity as listed in Table 2.

Table 2. Impact of methanol ambient concentration on the Reynolds number at $\left(P_{a m b}=106\right.$ bar, $\left.T_{a m b}=1200 \mathrm{~K}\right)$

\begin{tabular}{c|c}
\hline$Y_{\mathrm{CH}_{3} \mathrm{OH}, \mathrm{amb}}$ & $R e_{d}$ \\
\hline 0 & 7.4 \\
0.2 & 9 \\
0.4 & 10.3 \\
0.6 & 11.9 \\
\hline
\end{tabular}

The impact of the methanol ambient concentration on the temporal evolution of the normalized squared equivalent droplet diameter and mean droplet temperature is depicted in Figure 2, where the mean droplet temperature is computed using a volume averaged formula as $\left[T_{d}=\frac{\sum_{\text {cells }} T_{\text {cell }} \alpha_{l} V_{\text {cell }}}{\sum_{\text {cells }} \alpha_{l} V_{\text {cell }}}\right]$. A reduction of the droplet lifetime and an increase in its evaporation rate with increasing the methanol ambient concentration can be observed (Figure 2a). The reduction in the droplet lifetime can be attributed to the relatively higher solubility of methanol in the liquid phase than that of nitrogen. Indeed, Figure 3 shows that, for the case of $\left(\mathrm{Y}_{\mathrm{CH}_{3} \mathrm{OH}}=0.4\right)$, the total mass fraction of methanol and nitrogen in the liquid phase is relatively higher than that of nitrogen in the case of $\left(\mathrm{Y}_{\mathrm{CH}_{3} \mathrm{OH}}=0\right)$, which is accompanied by a higher evaporation rate of the $n$-dodecane droplet, as illustrated by the vaporous $n$-dodecane temporal evolution in Figure 4. Besides, the increase of the Reynolds number (Table 2) enhances the mixing and the evaporation processes leading to higher droplet evaporation rate and shorter lifetime as well as relatively higher droplet deformation for the case of $\left(Y_{\mathrm{CH}_{3} \mathrm{OH}}=0.4\right)$ (see Figure 3). 


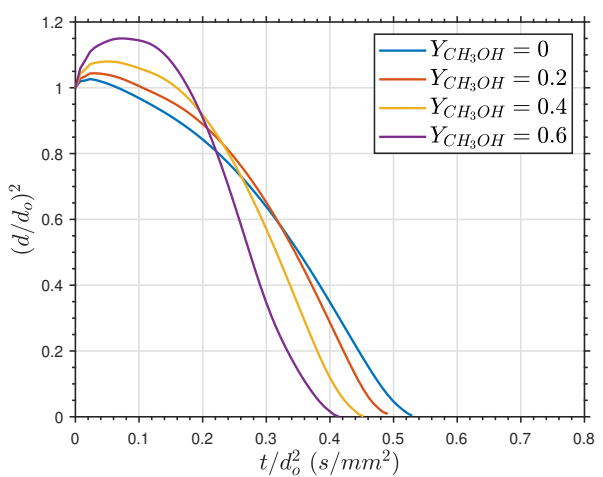

(a)

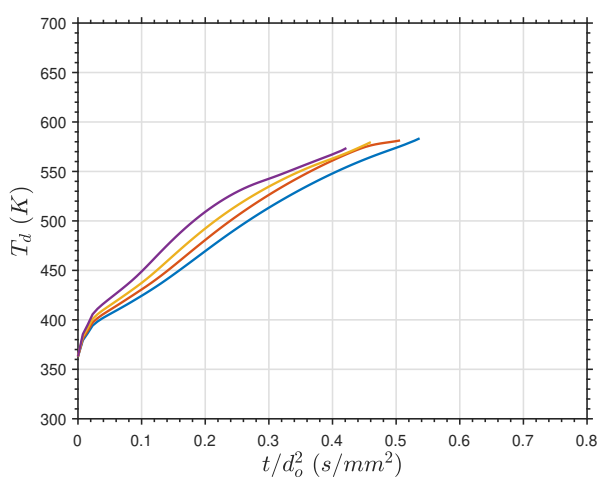

(b)

Figure 2. (a) Numerical prediction of the temporal evolution of the normalized squared equivalent droplet diameter and (b) mean droplet temperature for case $1\left(P_{a m b}=106 \mathrm{bar}, T_{a m b}=1200 \mathrm{~K}\right)$ with different initial methanol ambient concentration.

The phenomenon of the initial increase of the droplet size can also be observed in Figure $2 \mathrm{a}$. This phenomenon is classically attributed to the initial thermal expansion of the liquid droplet, as revealed in previous studies [28]. In addition, the current results show a relatively higher augmentation in the droplet size with increasing the methanol ambient concentration. This behavior can be related to the higher solubility of methanol in the liquid phase compared to nitrogen (see Figure 3), leading to a higher initial increase in the droplet size.

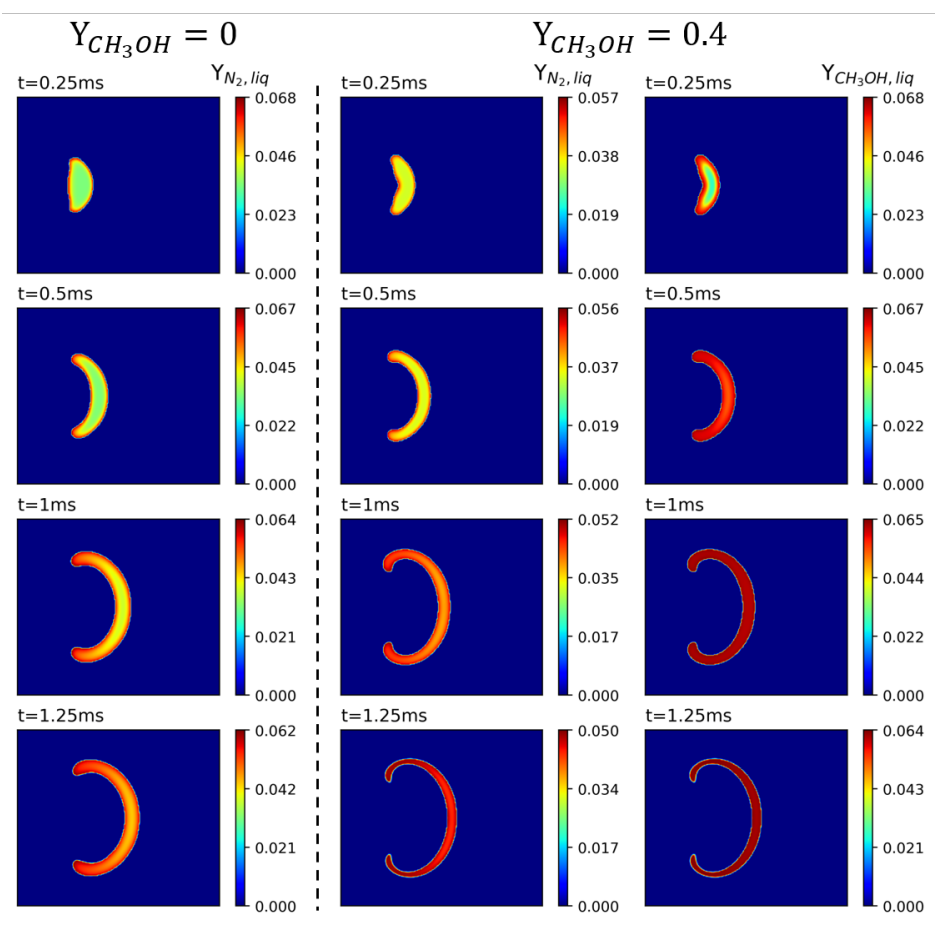

Figure 3. Temporal evolution of nitrogen and methanol mass fractions in the liquid phase for $Y_{\mathrm{CH}_{3} O H, a m b}=0$ (left) and $Y_{\mathrm{CH}_{3} \mathrm{OH}, \mathrm{amb}}=0.4$ (right) for case $1\left(P_{a m b}=106 \mathrm{bar}, T_{a m b}=1200 \mathrm{~K}\right)$. The droplet moves from left to right.

Moreover, Figure $2 \mathrm{~b}$ shows that the mean droplet temperature keeps rising until the end of the droplet lifetime without reaching constant temperature (wet-bulb temperature) for the various methanol ambient concentrations considered. This behavior has been observed in previous research [29, 30] when the ambient pressure is relatively high or supercritical with respect to the pure fuel critical point. It has been attributed to the simultaneous occurrence of the heating up and vaporization process due to the relatively low latent heat contrary to the lowpressure conditions, where the droplet heating process takes place almost separately from the vaporization process. 
Besides, Figure $2 \mathrm{~b}$ shows that at the end of the droplet lifetime, the mean droplet temperature reaches almost the same value independently of the methanol ambient concentration. However, the mean droplet temperature is relatively increasing for higher methanol ambient concentrations, which indicates a variation in the thermodynamic path followed by the droplet during the evaporation process.

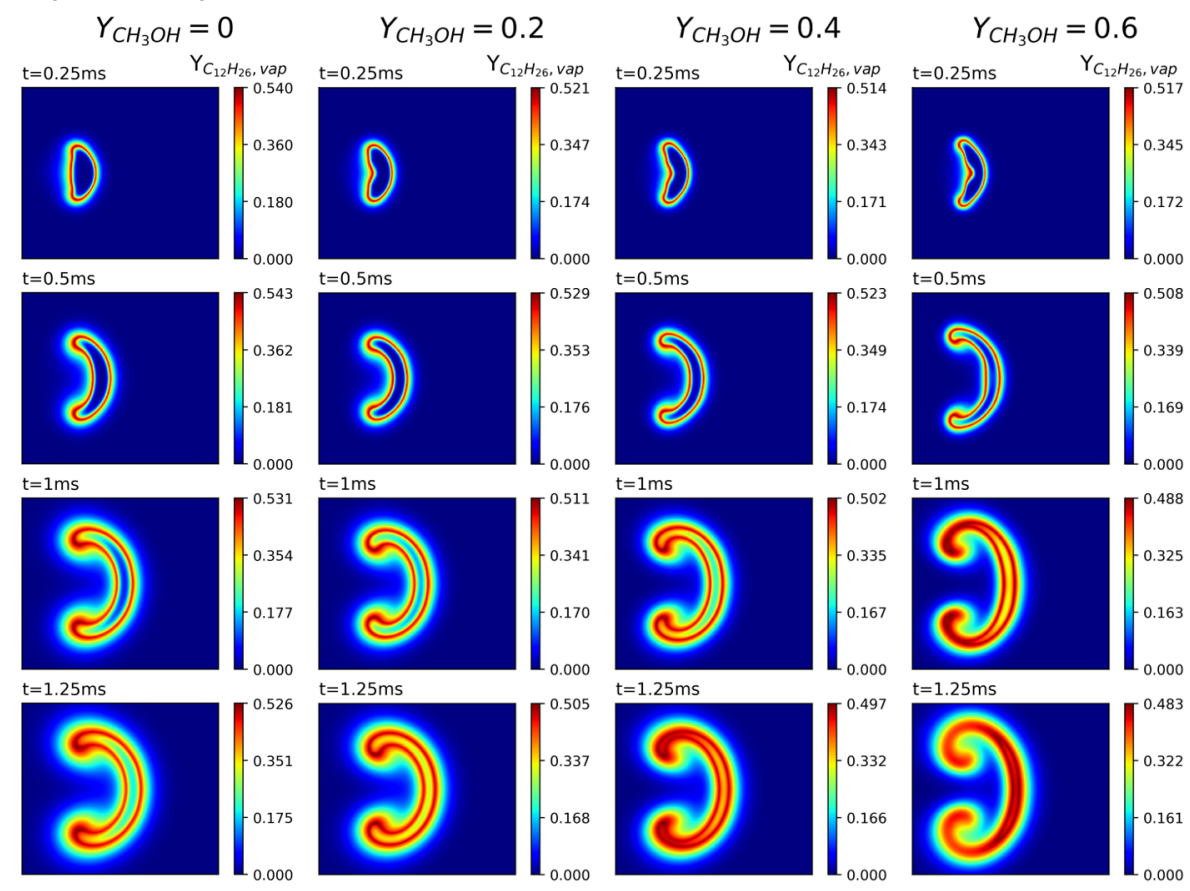

Figure 4. Temporal evolution of vaporous $\mathrm{n}$-dodecance mass fraction with various methanol ambient concentration for case $1\left(P_{a m b}=106 \mathrm{bar}, T_{a m b}=1200 \mathrm{~K}\right)$. The droplet moves from left to right.

For case $2\left(P_{a m b}=88 \mathrm{bar}, T_{a m b}=1000 \mathrm{~K}\right)$, similar trends as case 1 were observed for the impact of the methanol ambient concentration on the droplet lifetime and evaporation rate. In order to summarize the obtained results, an average evaporation rate constant $(k)$ was estimated as the slope of the almost linear part of the non-dimensional squared droplet diameter temporal evolution curve in the range $\left(\left(d / d_{0}\right)^{2}=0.2-0.8\right)$, using the least-squares linear fitting method and the intersection of the fitting line with the abscissa is regarded as the total evaporation time $\left(t_{L}\right)$. Figure 5 shows that for a constant methanol ambient concentration, the reduction of the ambient pressure and temperature is accompanied by a decrease in the estimated evaporation rate constant and an increase in the droplet lifetime.

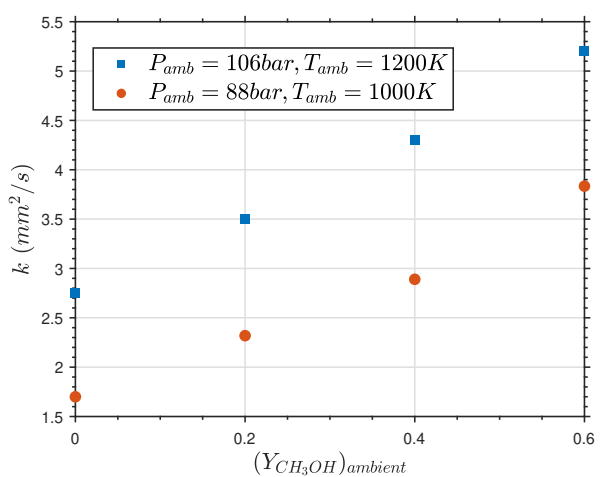

(a)



(b)

Figure 5. (a) Evaporation rate constant and (b) Normalized evaporation lifetime variation with ambient methanol concentration at different ambient temperature and pressure.

Finally, it is worth noting that, for all the test cases considered, the n-dodecane droplet initially in single liquid-phase state transits to a two-phase thermodynamic state for most of its lifetime, even if the ambient conditions considered are higher than the critical point of pure n-dodecane. 


\section{Conclusions}

The current work presented an extension of the RFM model [32] to handle ternary mixtures relevant to dual-fuel conditions, based on 4D tables generated by the IFPEN-Carnot thermodynamic library. The model has been applied to investigate $n$-dodecane droplet evaporation in bi-component ambient (nitrogen+methanol) using the CPA EOS. The main findings are:

1- The considered moving $n$-dodecane droplets exhibit a higher evaporation rate and a shorter lifetime as the methanol ambient concentration increases.

2- The ambient gas solubility in the liquid phase cannot be neglected due to its significant impact on the droplet evaporation as demonstrated by the current study, especially at the high ambient pressures considered.

3- The extended tabulated thermodynamic approach to ternary mixtures has proved to be an efficient remedy to the direct evaluation of costly phase equilibrium solver during the simulation.

\section{Acknowledgements}

This project has received funding from the European Union Horizon 2020 Research and Innovation programme. Grant Agreement No 861002 for the EDEM project.

\section{References}

[1] Yang, S., Yi, P., Habchi, C., 2020, International Journal of Multiphase Flow, 122:103145.

[2] Yi, P., Yang, S., Habchi, C., Lugo, R., 2019, Physics of Fluids, 31(2):026102.

[3] Sazhin, S. S., 2006, Progress in Energy and Combustion Science, 32(2):162-214.

[4] Saurel, R., Boivin, P., Le Métayer, O., 2016, Computers \& Fluids, 128:53-64.

[5] Jofre, L. and Urzay, J., 2021, Progress in Energy and Combustion Science, 82:100877.

[6] Ebrahimian, V. and Habchi, C., 2011, International Journal of Heat and Mass Transfer, 54(15-16):3552-3565

[7] Saurel, R., Abgrall, R., 1999, Journal of Computational Physics, 150(2):425-467.

[8] Pedrozo, V. B., May, I., Guan, W., Zhao, H., 2018, Fuel, 230:440-451.

[9] Miller, R. S., Harstad, K., Bellan, J., 1998, International Journal of Multiphase Flow, 24(6):1025-1055.

[10] Matheis, J., Hickel, S., 2018, International Journal of Multiphase Flow, 99:294-311.

[11] Le Ning, Duan, Q., Chen, Z., Kou, H., Liu, B., Yang, B., Zeng, K., 2020, Fuel,266:117034.

[12] Koukouvinis, P., Vidal-Roncero, A., Rodriguez, C., Gavaises, M., Pickett, L., 2020, Fuel, 275:117871.

[13] Kapila, A. K., Menikoff, R., Bdzil, J. B., Son, S. F., Stewart, D. S, Physics of Fluids, 13(10):3002-3024.

[14] Habchi, C., Dumont, N., and Simonin, O., 2008, 2020, Atomization and Sprays, 129-162.

[15] Habchi, C., 2015, Atomization and Sprays, 25(4):317-334.

[16] Dong, Y., Kaario, O., Hassan, G., Ranta, O., Larmi, M., Johansson, B., 2020, Fuel, $277: 117932$.

[17] Crua, C., Manin, J., Pickett, L. M., 2017, Fuel, 208:535-548.

[18] Chiapolino, A., Boivin, P., Saurel, R., 2017, Computers \& Fluids, 150:31-45.

[19] Bellan, J., 2000, Progress in Energy and Combustion Science, 26(4-6):329-366

[20] Baer, M. R., Nunziato, J. W., 1986, International Journal of Multiphase Flow, 12(6):861-889.

[21] Chung, T. H., Ajlan, M., Lee, L. L., Starling, K. E., 1988, Industrial \& Engineering Chemistry Research, 27(4):671-679.

[22] Michelsen, M. L., 1982, Fluid Phase Equilibria, 9(1):21-40.

[23] Peng, D. Y., Robinson, D. B., 1976, Industrial \& Engineering Chemistry Fundamentals, 15(1):59-64.

[24] Kontogeorgis, G. M., Voutsas, E. C., Yakoumis, I. V., Tassios, D. P., 1996, Industrial \& Engineering Chemistry Research, 35(11):4310-4318.

[25] Richards, K. J., Senecal,P. K., Pomraning, E. Converge 3.0.

[26] Ware, C., Knight, W., and Wells, D., 1991, Computers \& Geosciences, 17(7):985-993.

[27] De Hemptinne, J. C., Mougin, P., Barreau, A., Ruffine, L., Tamouza, S., and Inchekel, R., 2006, Oil \& Gas Science and Technology-Rev.IFP, 61(3):363-386.

[28] Nomura, H., Murakoshi, T., Suganuma, Y., Ujiie, Y., Hashimoto, N., Nishida, H., 2017, Proceedings of the Combustion Institute, 36(2):2425-2432.

[29] Jia, H., Gogos, G., 1992, Journal of Thermophysics and Heat Transfer, 6(4):738-745.

[30] Jia, H., Gogos, G., 1994, Acta Astronautica, 32(2):121-129.

[31] Karim, G. A., 2015, "Dual-fuel diesel engines". CRC Press.

[32] Yi, P., Jafari, S., Yang, S., Habchi, C., Sep. 2.-4. 2019, 29th European Conference on Liquid Atomization and Spray Systems.

[33] Engine Combustion Network (ECN), http://ecn.sandia.gov/ 